\title{
A Numerical quadrature for the Schwarz-Chimera Method
}

\author{
J. -B. Apoung Kamga ${ }^{1}$ and Olivier Pironneau ${ }^{2}$ \\ ${ }^{1}$ Laboratoire J.L. Lions, Université Paris VI apoung@ann.jussieu.fr \\ 2 and Institut Universitaire de france Curie Olivier.Pironneau@upmc.fr
}

\begin{abstract}
Chimera [10] happens to be a version of Schwarz' method and of Lions' space decomposition method (SDM). It was analyzed by Brezzi et al [1] but an estimate was missing for for numerical quadrature. We give it here with new numerical tests.
\end{abstract}

\section{Introduction}

Consider a Hilbert space $V$, a continuous bilinear form $a(u, \hat{u})$ symmetric with a coercivity constant $\alpha>0$ and $f$ regular for well posedness of

$$
a(u, \hat{u})=(f, \hat{u}) \forall \hat{u} \in V
$$

We assume that $V=V_{1}+V_{2} \quad V_{1} \cap V_{2}$ of non zero measure (i.e. overlapping) where each $V_{i}$ is a closed subspace of $V$. We will need also two continuous symmetric bilinear forms $b_{i}(u, \hat{u}), i=1,2$ coervice enough so that

$$
\sum_{1}^{2} b_{i}\left(\hat{u}_{i}\right)+a\left(\hat{u}_{i}\right) \geq a\left(\sum_{1}^{2} \hat{u}_{i}\right) \quad \forall \hat{u}_{i} \in V_{i}
$$

A typical example is the Dirichlet problem for $-\Delta u=f$ in $\Omega=\Omega_{1} \cup \Omega_{2}$ and such that $\Omega_{1} \cap \Omega_{2} \neq \emptyset$; denote by $S_{i}=\partial \Omega_{i} \cap \Omega_{j}, j \neq i$. Then set

$$
V_{i}=\left\{v \in L^{2}(\Omega):\left.\quad v\right|_{\Omega_{i}} \in V\left(\Omega_{i}\right),\left.\quad v\right|_{\Omega-\Omega_{i}}=0\right\}
$$

Algorithm 1 (Schwarz)

Begin loop with a Chosen $v_{i}^{0} \in V_{i}$, and $n=0$.

Find $v_{i}^{n+1}$ such that $v_{i}^{n+1}-v_{j}^{n} \in V_{i}, i, j=1,2, j \neq i$ by solving

$$
a\left(v_{i}^{n+1}, \hat{v}_{i}\right)=\left(f, \hat{v}_{i}\right) \quad \forall \hat{v}_{i} \in V_{i}
$$




\section{End loop}

The convergence has been analyzed by P.L. Lions[6] in a general setting. In search for precision, we present the following alternative; it uses $b_{i}(u, v)=b(u, v)=$ $(\beta u, v), i=1,2$ for some positive scalar $\beta$ and two arbitrary functions $u_{i}^{0} \in V_{i}$.

\section{Algorithm 2 (SDM)}

Begin loop with $n=0$ :

Find $u_{i}^{n+1} \in V_{i}$ by solving

$$
\begin{aligned}
& b\left(u_{1}^{n+1}-u_{1}^{n}, \hat{u}_{1}\right)+a\left(u_{1}^{n+1}+u_{2}^{n}, \hat{u_{1}}\right)=\left(f, \hat{u_{1}}\right) \quad \forall \hat{u_{1}} \in V_{1} \\
& b\left(u_{2}^{n+1}-u_{2}^{n}, \hat{u}_{2}\right)+a\left(u_{1}^{n}+u_{2}^{n+1}, \hat{u_{2}}\right)=\left(f, \hat{u_{2}}\right) \quad \forall \hat{u_{2}} \in V_{2}
\end{aligned}
$$

End loop

When $\beta=0$ Algorithm 2 is identical to Algorithm 1 with $u_{i}^{n+1}=v_{i}^{n+1}-v_{j}^{n}, i, j=$ $1,2, j \neq i$. If the decomposition is done with $m$ members with $m \geq 2$ then $u^{n+1}$ is found by solving

$$
b\left(u_{i}^{n+1}-u_{i}^{n}, \hat{u}_{i}\right)+a\left(u_{i}^{n+1}-u_{i}^{n}+\sum_{j=1}^{m} u_{j}^{n}, \hat{u}_{i}\right)=\left(f, \hat{u}_{i}\right) \quad \forall \hat{u}_{i} \in V_{i}
$$

Theorem 1. (J.L. Lions[4]) We assume (1-2). Then Algorithm (6) is convergent in the following sense: as $n \rightarrow \infty, u_{i}^{n} \rightarrow u_{i}^{*}$ with $u_{1}^{*}+u_{2}^{*}=u$ solution of $(1)$ and the decomposition is uniquely defined by

$$
\begin{aligned}
& (\beta+A) u_{1}=\frac{1}{2}(\beta+A)\left(u+u_{1}^{0}-u_{2}^{0}\right) \text { in } \Omega_{1} \cap \Omega_{2},\left.\quad u_{1}\right|_{S_{1}}=0,\left.\quad u_{1}\right|_{S_{2}}=u \\
& (\beta+A) u_{2}=\frac{1}{2}(\beta+A)\left(u+u_{2}^{0}-u_{1}^{0}\right) \text { in } \Omega_{1} \cap \Omega_{2},\left.\quad u_{2}\right|_{S_{2}}=0,\left.\quad u_{2}\right|_{S_{1}}=u \\
& A u_{i}=f \text { in } \Omega_{i} \backslash \Omega_{1} \cap \Omega_{2},\left.\quad u_{i}\right|_{\partial \Omega_{i}}=0
\end{aligned}
$$

\section{Discretization}

Let $\mathcal{T}_{1 h}\left(\operatorname{resp} \mathcal{T}_{2 h}\right)$ be a triangulation of $\Omega_{1}$ (resp $\left.\Omega_{2}\right)$, quasi-uniform [2], in the sense that, if $h_{M}$ and $h_{m}$ are the maximum and minimum edges in $\mathcal{T}_{1 h}$, and $H_{M}$ and $H_{m}$ are the maximum and minimum edges in $\mathcal{T}_{2 h}$, then there exists two constants $C_{1 T}$ and $C_{2 T}$ such that $h_{M} \leq C_{1 T} h_{m} \quad H_{M} \leq C_{2 T} H_{m}$. Without loss of generality we can also assume, that $h_{M} \leq H_{M}$. For clarity we assume that the $\Omega_{i}$ are polygonal and that $a($,$) is the Laplace operator with Dirichlet conditions. Let V_{1 h}$ and $V_{2 h}$ be two Lagrange conforming continuous finite element approximation spaces of order $p$ of $V_{1}=H_{0}^{1}\left(\Omega_{1}\right)$ and $V_{2}=H_{0}^{1}\left(\Omega_{2}\right)$. Then the discrete version of Algorithm 2 is to find for $\mathrm{i}=1,2, u_{i h}^{n+1} \in V_{i h}$ such that $\forall v_{i h} \in V_{i h}$

$$
\int_{\Omega_{i}}\left(\beta\left(u_{i h}^{n+1}-u_{i h}^{n}\right) v_{i h}+\nabla\left(u_{1 h}^{n+1}+u_{2 h}^{n}\right) \nabla v_{i h}\right)=\int_{\Omega_{i}} f v_{i h},
$$


Theorem 2. (see Hecht et al. [4]) Assume that the solution of (1) is in $H^{p+1}(\Omega)$ for some $p \geq 1$. Assume that in (7) $\left.u_{i}\right|_{\Omega_{i}} \in H^{p+1}\left(\Omega_{i}\right)$. If $u_{h}=\lim \left(u_{1 h}^{n}+u_{2 h}^{n}\right)$ is computed with Lagrange conforming finite elements of order $p$, then

$$
\left\|u-u_{h}\right\|_{1, \Omega} \leq C h^{p}\left(\left\|u_{1}\right\|_{p+1, \Omega_{1}}+\left\|u_{2}\right\|_{p+1, \Omega_{2}}\right) .
$$

\section{Numerical Quadrature}

As such, the scheme is too costly to implement because it requires the intersection of triangulations. Recall that the quadrature formula with integration points at the vertices is exact for polynomials of degree less than or equal to one. In particular, for a given triangle $\hat{T}$ one has

$$
\int_{\hat{T}} g d x d y=\frac{|\hat{T}|}{3} \sum_{i=1,2,3} g\left(q_{i}\right) \quad \forall g \in P_{1}(\hat{T}) .
$$

Hence we introduce the following quadrature rule.

$$
\begin{aligned}
(\nabla u, \nabla v)_{h} & :=\left.\sum_{T \in \mathcal{T}_{1 h}} \frac{|T|}{3} \sum_{i=1,2,3} \frac{\nabla\left(u_{\mid T}\right) \cdot \nabla v}{I_{\Omega_{1}+I_{\Omega_{2}}}}\right|_{q_{i}(T)} \\
& +\left.\sum_{K \in \mathcal{T}_{2 h}} \frac{|K|}{3} \sum_{j=1,2,3} \frac{\nabla\left(v_{\mid K}\right) \cdot \nabla u}{I_{\Omega_{1}}+I_{\Omega_{2}}}\right|_{q_{j}(K)} .
\end{aligned}
$$

where $I_{\Omega_{j}}(x)=1$ if $x \in \Omega_{j}$ and zero otherwise $(j=1,2)$. The notation $\nabla\left(u_{\mid T}\right)$ is used to indicate that we first restrict the function $u$ to $T$, and then we compute its gradient (which is actually constant in $T$ ). A similar interpretation holds for $\nabla\left(v_{\mid K}\right)$. With such definitions we propose to solve the discrete problems:

- Find $u_{i h}^{n+1} \in V_{i h}$ such that $\forall v_{i h} \in V_{i h}$

$$
\begin{aligned}
& b\left(u_{1 h}^{n+1}-u_{1 h}^{n}, \hat{u}_{1 h}\right)+a_{h}\left(u_{1 h}^{n+1}+u_{2 h}^{n}, \hat{u}_{1 h}\right)=\left(f, \hat{u}_{1 h}\right) \quad \forall \hat{u}_{1 h} \in V_{1 h} \\
& b\left(u_{2 h}^{n+1}-u_{2 h}^{n}, \hat{u}_{2 h}\right)+a_{h}\left(u_{1 h}^{n}+u_{2 h}^{n+1}, \hat{u}_{2 h}\right)=\left(f, \hat{u}_{2 h}\right) \quad \forall \hat{u}_{2 h} \in V_{2 h}
\end{aligned}
$$

Clearly these define $u_{i h}^{n+1}$ uniquely. At convergence the problem solved is

- Find $u_{i h} \in V_{i h}$ such that $\forall \hat{u}_{i h} \in V_{i h}$

$$
a_{h}\left(u_{1 h}+u_{2 h}, \hat{u}_{1 h}+\hat{u}_{2 h}\right)=\left(f, \hat{u}_{1 h}+\hat{u}_{2 h}\right) .
$$

Under a mild assumption on the triangulations this discrete problem has a unique solution at least when linear elements are used $(p=1)$ :

each vertex of $\mathcal{T}_{1 h}$ is internal to a triangle $K$ of $\mathcal{T}_{2 h}$, and conversely.

This is because of the coercivity of the bilinear form and of the uniqueness of the decomposition $u_{h}=u_{1 h}+u_{2 h}$ :

Theorem 3. (Brezzi)

Assume (14) holds. If two functions $u_{i h} \in V_{i h}, i=1,2$ coincide on a connected subset $\mathcal{X}$ of $\Omega_{1} \cap \Omega_{2}$, then both $u_{\text {ih }}$ are linear (not just piecewise linear) in $\mathcal{X}$. Furthermore

$$
a_{h}\left(u_{1 h}+u_{2 h}, u_{1 h}+u_{2 h}\right) \geq c\left\|u_{1 h}+u_{2 h}\right\|^{2} \text { for all } u_{i h} \in V_{i h}
$$


One more property is needed, the continuity of $a_{h}$, and then we can apply Strang's lemma and obtain the following estimate:

Proposition 1. (see [4]) Assume that the triangulations of $\Omega_{1}$ and $\Omega_{2}$ are compatible in the sense that they give a coercive bilinear form. Assume that $a_{h}$ is uniformly continuous for all $h$. Then the error between the approximate problem (13) and the continuous problem is

$$
\left\|u-u_{h}\right\|<C h\left(\left\|u_{1}\right\|_{2, \Omega_{1}}+\left\|u_{2}\right\|_{2, \Omega_{2}}\right)
$$

\section{Continuity of the Approximate Bilinear Form}

\subsection{The One Dimensional Case}

We begin with the mono dimensional case because the proof is easier to follow. The same argument will be extended to $2 \mathrm{D}$.

Proposition 2. In one dimension the constant of continuity $C$ in

$$
\begin{aligned}
& \left|\nabla u_{H}+\nabla u_{h}\right|_{h} \leq C\left|\nabla u_{H}+\nabla u_{h}\right| \\
& \text { satisfies } \quad C^{2} \leq \frac{1}{2} \max \left\{\max _{i \in K} \frac{\left|x_{i+1}-x_{i}\right|}{\left|x_{i}-X_{j(i)}\right|}, \max _{i \in L} \frac{\left|X_{i+1}-X_{i}\right|}{\left|X_{i}-x_{j(i)}\right|}\right\}
\end{aligned}
$$

where $K$ (resp. $L)$ is the set of $i$ such that $j(i)$ exists with $X_{j(i)} \in\left[x_{i}, x_{i+1}\right]$ (resp $\left.x_{j(i)} \in\left[X_{i}, X_{i+1}\right]\right)$. Consequently $C$ is bounded by the square root of half the largest interval length divided by the smallest distance between two vertices.

Proof For any real valued function $f$,

$$
\max _{u_{h}, u_{H}} f\left(\nabla u_{H}+\nabla u_{h}\right) \leq \max _{U_{h}, U_{H}} f\left(U_{H}+U_{h}\right)
$$

where $u_{h}, u_{H}$ are real valued continuous- piecewise linear functions on their mesh and $U_{H}, U_{h}$ are piecewise constant vector valued on their meshes, because every $\nabla u$ is a $U$ and the opposite is not true when boundary conditions exist at both ends.

Denote $V=U_{H}+U_{h}$. As $V$ is piecewise constant, by definition

$$
\begin{aligned}
& 4|V|_{h}^{2}=\sum_{i}\left|x_{i+1}-x_{i}\right|\left(|V|\left(x_{i}^{+}\right)^{2}+|V|\left(x_{i+1}^{-}\right)^{2}\right)+\sum_{j}\left|X_{j+1}-X_{j}\right|\left(|V|\left(X_{j}^{+}\right)^{2}+|V|\left(X_{j+1}^{-}\right)^{2}\right) \\
& 2|V|_{0}^{2}=\sum_{i, j \in K}\left|X_{j}-x_{i}\right|\left(|V|\left(X_{j}^{-}\right)^{2}+|V|\left(x_{i}^{+}\right)^{2}\right)+\sum_{i, j \in L}\left|x_{i}-X_{j}\right|\left(|V|\left(X_{j}^{+}\right)^{2}+|V|\left(x_{i}^{-}\right)^{2}\right) \\
& \quad+\sum_{i \in I}\left|x_{i+1}-x_{i}\right|\left(|V|\left(x_{i}^{+}\right)^{2}+|V|\left(x_{i+1}^{-}\right)^{2}\right)+\sum_{j \in J}\left|X_{j+1}-X_{j}\right|\left(|V|\left(X_{j}^{+}\right)^{2}+|V|\left(X_{j+1}^{-}\right)^{2}\right)
\end{aligned}
$$

where $I, J$ are the set of intervals completely inside an interval of the other mesh, i.e.

$$
I=\left\{i: \exists j \text { s.t. }\left[x_{i}, x_{i+1}\right] \subset\left[X_{j}, X_{j+1}\right\} \quad J=\left\{j: \exists i \text { s.t. }\left[X_{j}, X_{j+1}\right] \subset\left[x_{i}, x_{i+1}\right]\right\}\right.
$$


Denote by $\mathrm{N}$ the set of values of $V_{k}$ of $V$ right or left of $x_{i}$ or $X_{j}$. As $f(V)=$ $|V|_{h}^{2} /|V|_{0}^{2}$ we see that it is of the type $f(V)=\sum_{k \in N} \alpha_{k}|V|_{k}^{2} / \sum_{k \in N} \beta_{k}|V|_{k}^{2}$ with $\alpha_{i}$ equal to a fourth of $x_{i+1}-x_{i}$ or $X_{i+1}-X_{i}$, and $\beta_{i}$ equal half of $x_{i+1}-x_{i}$ or $X_{i+1}-X_{i}$ or $x_{i}-X_{j(i)}$ or $X_{i}-x_{j(i)}$ a sum of two of those. Of course it is important to notice that all values appear both in the nominator and denominator. With a change of variable this is also

$$
f(W)=\frac{\sum \frac{\alpha_{k}}{\beta_{k}} W_{k}^{2}}{\sum W_{k}^{2}} . \text { Then } \quad \max f(W)=\max _{k} \frac{\alpha_{k}}{\beta_{k}}
$$

Now that this is established we can answer much simply the problem of finding $\max \alpha_{k} / \beta_{k}$ : it is the largest ratio of coefficients multiplying $V\left(x_{i}^{ \pm}\right)$or $V\left(X_{j}^{ \pm}\right)$in the expressions for $|V|_{h}$ and $|V|_{0}^{2}$, i.e. in (16).

\subsection{The Two Dimensional Case}

A similar argument applies in two dimensions. Assume we have two triangulations with triangles $\left\{T_{k}\right\}_{1}^{N}$ and $\left\{t_{k}\right\}_{1}^{n}$ respectively and vertices $Q_{i}$ and $q_{i}$. Recall that

$$
|V|_{h}^{2}=\frac{1}{6} \sum_{k=1}^{N} \sum_{j=1,2,3} \mid V_{T_{k}}\left(\left.Q_{i_{j}}\right|^{2}\left|T_{k}\right|+\frac{1}{6} \sum_{k=1}^{n} \sum_{j=1,2,3} \mid V_{t_{k}}\left(\left.q_{i_{j}}\right|^{2}\left|t_{k}\right|\right.\right.
$$

where $i_{j}, j=1,2,3$ are the numbers of the 3 vertices for each triangle. On the other hand the exact value $|V|_{0}^{2}$ is

$$
|V|_{0}^{2}=\sum_{k, l} \sum_{j=1,2,3}\left|V_{R_{k l}}\left(\xi_{k l}\right)\right|^{2}\left|R_{k l}\right|
$$

where $R_{k l}=T_{k} \cap t_{l}$ and $\xi_{k l}$ is any point in $R_{k l}$.

For each $Q_{i_{j}}\left(\right.$ resp $q_{i_{j}}$ ) in (17) there is a $R_{k l}$ which contains it. For these $R$ let us choose in (18) $\xi_{k l}=Q_{i_{j}}$ and $q_{i_{j}}$. Then for every term in $|V|_{h}^{2}$ there is a corresponding term in $|V|_{0}^{2}$ :

$$
\frac{1}{6} \mid V_{T_{k}}\left(\left.Q_{i_{j}}\right|^{2}\left|T_{k}\right| \text { in correspondence with } \mid V_{T_{k}}\left(\left.Q_{i_{j}}\right|^{2}\left|T_{k} \cap t_{l}\right|\right.\right.
$$

where $l$ is such that $Q_{i_{j}} \in t_{l}$; and similarly with $q_{i_{j}}$.

However in this construction we will assign as many $\xi$ to a $R$ as the number of vertices it contains. So the safest is to divide by 3 the second term in (19).

Notice that some $R$ do not contain any vertex; if we leave these aside we obtain

$$
\frac{|V|_{h}^{2}}{|V|_{0}^{2}} \leq \frac{1}{2} \max _{k, l}\left\{\frac{\max \left\{\left|T_{k}\right|,\left|t_{l}\right|\right\}}{\left|T_{k} \cap t_{l}\right|}: T_{k} \cap t_{l} \text { contains at least one vertex }\right\}
$$

So we have proved the following 
Proposition 3. In two dimensions, the constant of continuity between the approximate norm $\left|\nabla u_{H}+\nabla u_{h}\right|_{h}$ and the exact one is proportional to the square root of the biggest ratio of area between a triangle $T$ and one of its polygons $T \cap t$ where $t$ is a triangle of the other triangulation containing a vertex of $T$.

The proof is similar, except that in the exact norm there are terms which do not exist in the approximate norm; but these are positive and appear in the denominator of the expression which bounds $\mathrm{C}$.

Remark 1. Consider the case where each triangle of the mesh $h$ has no more than one vertex of the mesh $H$ inside. Assume that this vertex is near the center of the triangle (or segment in one-D). Assume that all angles between two intersecting edges are bounded away from 0 and $\pi$ when $h, H \rightarrow 0$ and that $H / h$ and $h / H$ do not tend to 0 . Then $C$ is strictly posivite in the limit. However it is difficult in practice to insure that no angle tend to zero when the mesh is refined

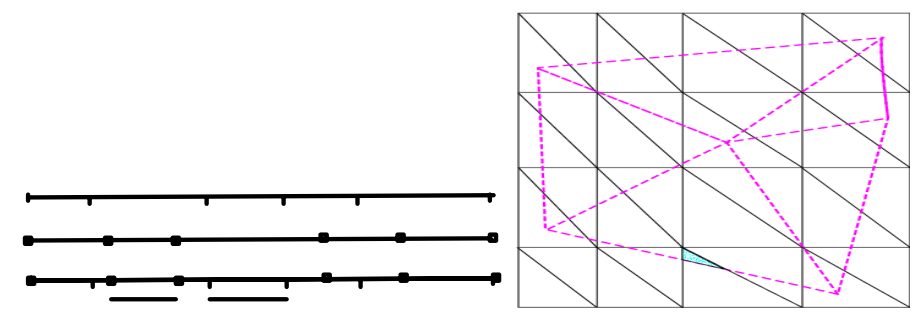

Fig. 1. Top: Two meshes in $1 \mathrm{D}$ and the intersected mesh. Two intervals have been singled out as they are strictly inside an interval of the other mesh; the continuity constant is proportional to the ratio of the smallest interval in the intersected mesh to the biggest interval in both meshes neighbor to that smallest one. Bottom: The continuity constant is proportional to the smallest polygon containing a vertex (shown with a texture) divided by the area of the biggest neighbor triangle in both meshes. Notice that some edges pass right through a vertex in this example, so if one mesh is shifted slightly the continuity constant estimate suddenly deteriorates.

\section{Numerical Test}

In all the numerical tests that follow, errors are evaluated on the intersected mesh, using exact quadrature formula. The problem to solve is $-\Delta u=f$ in $\Omega, u=$ $g$ on $\partial \Omega$. Data are chosen so that $u(x, y)=\sin (x) \times \sin (y)$.

\subsection{Exact quadrature}

This formula is introduced so as to give an exact computation for integral like $\int_{T_{h} \cap T_{H}} \Phi \Psi$. Where $\Phi$ and $\Psi$ see FIG.2 below are P1-lagrange functions on the 


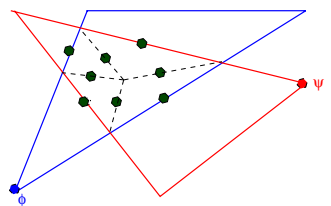

Fig. 2. Quadrature points for exact evaluation of $\int_{T_{h} \cap T_{H}} \Phi \Psi$.

triangle $T_{h}$ and $T_{H}$ respectively. It is based on the intersection of the two meshes. $\Omega_{1}$ is a circle of radius 1 centered at $(0,0)$ and $\Omega_{2}$ is the square $(-0.5,0.5)^{2} . \Omega_{2}$ is going to be meshed with uniform triangles so that by diadic refinement, order of convergence should be easily evaluated see Table1.

\subsection{First quadrature formula}

Table1 displays the results when(11) is used. Notice that by taking $u \in V_{h}, v \in V_{h}$, we don't recover the ordinary approximated bilinear form for the Laplace equation on the domain $\Omega_{1}$. So for a parallel implementation of (12), instead, we must find $u^{n+1} \in V_{0 h}$ such that (here $\left.b \equiv 0\right), \forall \hat{v} \in V_{0 h}\left(\Omega_{1}\right)$

$$
\left(\nabla u_{1}^{n+1}, \nabla \hat{v}\right)_{h}=(f, \hat{v})-\left(\nabla u_{2}^{n}, \nabla \hat{v}\right)_{h H}-\frac{1}{2}\left(\nabla u_{1}^{n}, \nabla \hat{v}\right)_{h}+\frac{1}{2}\left(\nabla u_{1}^{n}, \nabla \hat{v}\right)_{H} .
$$

Here $(., .)_{h},(., .)_{H}$ don't need quadrature. For the numerical experiments, we have taken $\Omega_{2}=(-2,3) \times(-3,2)$ and $\Omega_{1}=\left(-\frac{4}{3}, \frac{5}{3}\right) \times\left(-\frac{5}{3}, \frac{4}{3}\right)$.

\subsection{Second quadrature formula}

On the way, we have also tried, for $u_{1}, v_{1} \in V_{h}, u_{2}, v_{2} \in V_{H}$

$$
\begin{aligned}
\left(\nabla u_{1}, \nabla v_{2}\right)_{h H, \Omega_{1} \cap \Omega_{2}} & :=\sum_{K \in \mathcal{K}_{H}} \frac{|K|}{3} \sum_{j=1,2,3}\left(\nabla\left(u_{1}\right) \cdot \nabla\left(\left.v_{2}\right|_{K}\right)\right)\left(q_{j}(K)\right) \\
\left(\nabla u_{2}, \nabla v_{1}\right)_{H h, \Omega_{1} \cap \Omega_{2}}: & =\sum_{T \in \tau_{h}} \frac{|T|}{3} \sum_{j=1,2,3}\left(\nabla\left(u_{2}\right) \cdot \nabla\left(\left.v_{1}\right|_{T}\right)\right)\left(q_{j}(T)\right) .
\end{aligned}
$$

\subsection{Schwarz algorithm with quadrature}

Finally, to compare with Schwarz' algoritm, let $\pi_{h H}: V_{h} \mapsto V_{H}$ and $\pi_{H h}: V_{H} \mapsto$ $V_{h}$ the $P^{1}$ interpolation operators. Then the Schwarz method is implemented as

$$
\left\{\begin{array}{l}
\left(\nabla\left(u^{n+1}+\pi_{H h} v^{n}\right), \nabla \hat{u}\right)_{h}=(f, \hat{u})_{h} \quad \forall \hat{u} \in V_{0 h} \\
\left(\nabla\left(v^{n+1}+\pi_{h H} u^{n}\right), \nabla \hat{v}\right)_{H}=(f, \hat{v})_{H} \quad \forall \hat{v} \in V_{0 H}
\end{array}\right.
$$




\begin{tabular}{|c|c|c|c|c|c|}
\hline & & \multicolumn{5}{|c|}{$u-\left(u_{1}+u_{2}\right)$} \\
\hline$N 1$ & $N 2$ & $L^{2}$ error & rate & $\nabla L^{2}$ error & rate \\
\hline \hline \multicolumn{7}{|c|}{ Exact Quadrature } \\
\hline 10 & 5 & $1.54 E-02$ & - & $2.25 E-01$ & - \\
\hline 20 & 10 & $3.78 E-03$ & 2.02 & $1.11 E-01$ & 1.02 \\
\hline 40 & 20 & $8.24 E-04$ & 2.2 & $5.03 E-02$ & 1.15 \\
\hline \multicolumn{7}{|c|}{ First Quadrature } \\
\hline 3 & 5 & $4.64 E-01$ & - & $1.00 E-00$ & - \\
\hline 6 & 10 & $8.18 E-02$ & 2.50 & $5.44 E-01$ & 0.89 \\
\hline
\end{tabular}

\begin{tabular}{|c|c|c|c|c|c|}
\hline & & \multicolumn{5}{|c|}{$u-\left(u_{1}+u_{2}\right)$} \\
\hline$N 1$ & $N 2$ & $L^{2}$ error & rate & $\nabla L^{2}$ error & rate \\
\hline \hline \multicolumn{7}{|c|}{ Second Quadrature } \\
\hline 10 & 5 & $1.85 E-02$ & - & $2.32 E-01$ & - \\
\hline 20 & 10 & $5.66 E-03$ & 1.71 & $1.16 E-01$ & 1.00 \\
\hline 40 & 20 & $1.03 E-03$ & 2.45 & $5.34 E-02$ & 1.12 \\
\hline \multicolumn{7}{|c|}{ Schwarz overlapping } \\
\hline 10 & 5 & $1.68 E-02$ & - & $2.29 E-01$ & - \\
\hline 20 & 10 & $3.49 E-03$ & 2.26 & $1.09 E-01$ & 1.06 \\
\hline 40 & 20 & $9.15 E-04$ & 1.93 & $5.13 E-02$ & 1.09 \\
\hline
\end{tabular}

Table 1. Numerical $L^{2}$ errors, and convergence rate, for P1 polynomials with different quadrature formula. $N_{i}, i=1,2$ is the number of vertices on the boundary of the domain $\Omega_{i}$.

\section{Conclusion}

The results show that the first quadrature formula has optimal errors numerically but the results are very sensitive to the position of the grid points. Good results are obtained with the second quadrature formula, which is also easy to implement in 3D but no error analysis are available yet.

\section{References}

1. Brezzi,F., Lions, J.L., Pironneau, O. (2001): Analysis of a Chimera Method. C.R.A.S., 332, 655-660

2. Ciarlet, Ph. G. (1978): The Finite Element Method. North Holland Amsterdam

3. Hecht, F., Lions, J.L., Pironneau, O. (to appear): Domain Decomposition Algorithm for Computed Aided Design. (anniversary book of Necas)

4. Hecht, F., Pironneau, O. (1999): Multiple meshes and the implementation of freefem+. INRIA report. Also on the web athttp://www.ann.jussieu.fr/pironneau.

5. Lions, J.L., Pironneau, O. (1998): Algorithmes parallèles pour la solution de problèmes aux limites. C.R.A.S., 327, 947-352

6. Lions, J.L., Pironneau, O. (1999): Domain decomposition methods for CAD. C.R.A.S., $32873-80$

7. Lions, J.L., Pironneau, O. (to appear): A Domain Decomposition Algorithm, C.R.A.S. (to appear).

8. Lions, P.L. $(1988,89,90)$ On the Schwarz alternating method, I,II,III. Int. Symposium on Domain decomposition Methods for Partial Differential Equations. SIAM Philadelphia

9. Lions, J.L., Magenes, E. (1968): Problèmes aux limites non-homogènes et applications, Vol 1. Dunod Paris

10. Steger J.L. (1991): The Chimera method of flow simulation. Workshop on applied CFD, Univ. of Tennessee Space Institute 


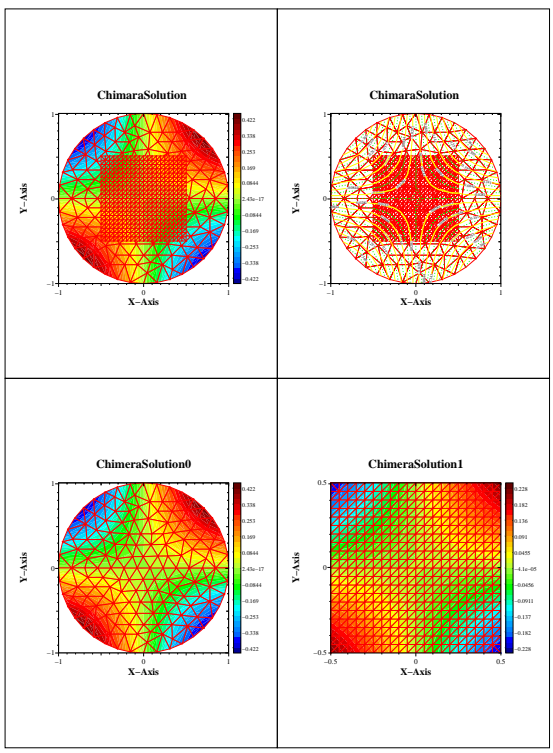

Fig. 3. Chimera solution of test case with exact quadrature formula. Bottom : solution on each subdomain.

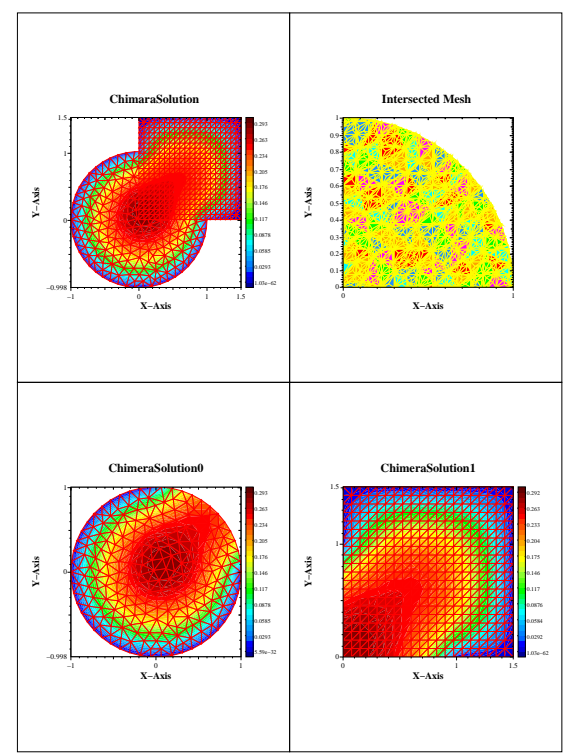

Fig. 4. Chimera solution of $(\Delta u=$ 1 on $\Omega, u=0$ on $\partial \Omega$ ) with second quadrature formula. Top right: intersected mesh. Bottom : solution on each subdomain. 